SISSA 77/2007/EP

INRNE-TH-07-12

\title{
Positive Energy Representations, Holomorphic Discrete Series and Finite-Dimensional Irreps
}

\author{
V.K. Dobrev \\ Scuola Internazionale Superiore di Studi Avanzati \\ via Beirut $2-4$ \\ 34014 Trieste, Italy \\ and \\ Institute for Nuclear Research and Nuclear Energy ${ }^{1}$ \\ Bulgarian Academy of Sciences \\ 72 Tsarigradsko Chaussee, 1784 Sofia, Bulgaria
}

\begin{abstract}
Let $G$ be a semi-simple non-compact Lie group with unitary lowest/highest weight representations. We consider explicitly the relation between three types of representations of $G$ : positive energy (unitary lowest weight) representations, (holomorphic) discrete series representations and non-unitary finite-dimensional irreps. We consider mainly the conformal groups $S O_{o}(n, 2)$ treating in full detail the cases $n=1,3,4$.
\end{abstract}

1 Permanent address. 


\section{Introduction}

Let $G$ be a semi-simple non-compact Lie group with unitary lowest/highest weight representations, i.e., $(G, K)$ is a Hermitian symmetric pair, where $K$ is a maximal compact subgroup of $G$ [1]. Let $\mathcal{G}$ be the Lie algebra of $G$. Then, $\mathcal{G}$ is one of the following Lie algebras: $s u(m, n), s o(n, 2), s p(2 n, R), s o^{*}(2 n), E_{6(-14)}, E_{7(-25)}[2]$. These groups/algebras have also discrete series representations, since $\operatorname{rank} G=\operatorname{rank} K$, and $G \supset K \supset H$, where $H$ is a Cartan subalgebra of $G[3]$.

In this paper we start a discussion on the relation between three types of representations of $G$ : positive energy (i.e., unitary lowest weight) representations, (holomorphic) discrete series representations and finite-dimensional representations (the latter are not unitary).

There are some general facts that are known about these relationships. For example every discrete series representation has the same infinitesimal character (Casimirs) as some finite-dimensional representation [3]. According to the results of [1],[3],[4] a holomorphic/antiholomorphic discrete series representation is (infinitesimally) equivalent to a unitary lowest/highest weight representation. The submerging of the set of discrete points enumerating the holomorphic discrete series in the semi-infinite interval parametrizing the unitary lowest weight representations is given in [4]. ${ }^{2}$ The embedding of discrete series representations into elementary representations ${ }^{3}$ (called also generalized principal series representations) is given in [5],[6],[7]. (Other pertinent references are [8],[9],[10],[11],[12],[13].) However, the latter two very important connections are not used simultaneously in the mathematical literature.

Our input in the discussion of these relationships may be summarized as follows. First of all, we use all relationships between the three types of representations. In particular, we use essentially the fact that discrete series representations and finite-dimensional representations occur as subrepresentations of elementary representations. The elementary representations in question are topologically reducible (and not unitary). We group the (reducible) ERs with the same Casimirs in sets called multiplets [14],[15]. The multiplet corresponding to fixed values of the Casimirs may be depicted as a connected graph, the vertices of which correspond to the reducible ERs and the lines between the vertices correspond to intertwining operators. ${ }^{4}$ The explicit parametrization of the multiplets and of their ERs is important for understanding of the situation. Especially important are the multiplets containing (as subrepresentation of some reducible ER of the multiplet) a finite-dimensional representation. Each such multiplet contains some discrete series representation(s) (as subrepresentation(s) of other reducible ER(s) of the multiplet), and all discrete series representations are contained in some such multiplet. Furthermore, from these multiplets - by certain limiting procedure - one can obtain all multiplets containing limits of discrete series. (The latter resulting multiplets do not contain finite-dimensional

2 Note that EHW [4] work in the conjugate picture with highest weight modules.

3 The precise definition with relevant references is given below.

4 For simplicity only the operators which are not compositions of other operators are depicted.

The ERs which are related by non-trivial intertwining operators are said to be partially equivalent. 
representations.) Finally, using the multiplets, we can identify the intertwining operators relevant for discrete series representations and the finite-dimensional representation.

We should also mention that in distinction to mathematicians, in our considerations we are using induction also from maximal parabolics that are not cuspidal (e.g., on the example of $\mathcal{G}=s o(n, 2)$ in this paper $)$.

In the present paper we start such a description by discussing in some detail the conformal case when $\mathcal{G}=s o(n, 2)$. There are two typical cases when $n>2: n$ odd and $n$ even. Furthermore, the case $n=1$ is special and is discussed separately. (The case $n=2$ is reduced to the case $n=1$.) Thus, the paper is organized as follows. In Section 2 we give the general setting. In Section 3 we specify the setting to the conformal case. In Sections $4,5,6$ we treat the cases $n=1,3,4$ in detail.

\section{Preliminaries}

Let $G$ be a semisimple non-compact Lie group, and $K$ a maximal compact subgroup of $G$. Then we have an Iwasawa decomposition $G=K A N$, where $A$ is abelian simply connected, a vector subgroup of $G, N$ is a nilpotent simply connected subgroup of $G$ preserved by the action of $A$. Further, let $M$ be the centralizer of $A$ in $K$. Then the subgroup $P_{0}=M A N$ is a minimal parabolic subgroup of $G$. A parabolic subgroup $P=M^{\prime} A^{\prime} N^{\prime}$ is any subgroup of $G$ (including $G$ itself) which contains a minimal parabolic subgroup. ${ }^{5}$

The importance of the parabolic subgroups comes from the fact that the representations induced from them generate all (admissible) irreducible representations of $G$ [17]. For the classification of all irreducible representations it is enough to use only the so-called cuspidal parabolic subgroups $P=M^{\prime} A^{\prime} N^{\prime}$, singled out by the condition that $\operatorname{rank} M^{\prime}=$ rank $M^{\prime} \cap K$ [18],[19], so that $M^{\prime}$ has discrete series representations [3]. However, often induction from non-cuspidal parabolics is also convenient and we shall use it below.

Let $\nu$ be a (non-unitary) character of $A^{\prime}, \nu \in \mathcal{A}^{*}$, let $\mu$ fix an irreducible representation $D^{\mu}$ of $M^{\prime}$ on a vector space $V_{\mu}$.

We call the induced representation $\chi=\operatorname{Ind}_{P}^{G}(\mu \otimes \nu \otimes 1)$ an elementary representation of $G[20]$. (These are called generalized principal series representations (or limits thereof) in [6].) Their spaces of functions are:

$$
\mathcal{C}_{\chi}=\left\{\mathcal{F} \in C^{\infty}\left(G, V_{\mu}\right) \mid \mathcal{F}(\text { gman })=e^{-\nu(H)} \cdot D^{\mu}\left(m^{-1}\right) \mathcal{F}(g)\right\}
$$

where $a=\exp (H) \in A^{\prime}, \quad H \in \mathcal{A}^{\prime}, m \in M^{\prime}, \quad n \in N^{\prime}$.

For our purposes we need to restrict to maximal parabolic subgroups $P$, (so that $\operatorname{rank} A^{\prime}=1$ ), that may not be cuspidal (the importance of such occurrences is explained on the example of $\mathcal{G}=s o(n, 2)$ below $)$.For the representations that we consider the character $\nu$ is parameterized by a real number $d$, called the conformal weight or energy (the latter for reasons that will become clear below).

5 The number of non-conjugate parabolic subgroups is $2^{r}$, where $r=\operatorname{rank} A$, cf., e.g., [16]. 
We restrict also to the case of finite-dimensional (nonunitary) representations $\mu$ of $M^{\prime}$.

An important ingredient in our considerations are the unitary lowest weight representations of $\mathcal{G}$. These can be realized as factor-modules of Verma modules $V^{\Lambda}$ over $\mathcal{G}^{\mathbb{C}}$, where $\Lambda \in\left(\mathcal{H}^{\mathscr{C}}\right)^{*}, \quad \mathcal{H}^{\mathbb{C}}$ is a Cartan subalgebra of $\mathcal{G}^{\mathbb{C}}$, the lowest weight $\Lambda=\Lambda(\chi)$ is determined uniquely from $\chi$ [15]. Unitarity means positivity w.r.t. the Shapovalov form in which the conjugation is the one singling out $\mathcal{G}$ from $\mathcal{G}^{\mathbb{C}}$.

Actually, since our ERs are induced from finite-dimensional representations of $\mathcal{M}^{\prime}$ the Verma modules are always reducible. Thus, it is more convenient to use generalized Verma modules $\tilde{V}^{\Lambda}$ such that the role of the lowest weight vector $v_{0}$ is taken by the finitedimensional space $V_{\mu} v_{0}$. For the generalized Verma modules (GVMs) the reducibility is controlled only by the value of the conformal weight $d$. Matters are arranged so that there is a real number $d_{0}$ called the first reduction point (FRP), such that for $d>d_{0}$ the GVMs are irreducible and unitary. For $d=d_{0}$ the GVM is reducible (in general) with invariant subspace $I^{\Lambda}$ so that the factor space $L_{\Lambda} \cong \tilde{V}^{\Lambda} / I^{\Lambda}$ is irreducible and unitary. For $d<d_{0}$ in some cases there is a discrete set of $d$-values for which the GVM is reducible and again the factor space $L_{\Lambda}$ is irreducible and unitary. This picture was known for the conformal cases $\mathcal{G}=s o(n, 2)$ when $n=3$ [21] (for more modern exposition cf. also [22]) and $n=4$ [23], but was established for all algebras with lowest/highest weight modules in $[4] .^{6}$

We turn now to the relation of discrete series representations with lowest weight modules.

The unitary lowest weight generalized Verma modules are infinitesimally equivalent to holomorphic discrete series when $d=d_{0}+k c_{0}, k=A\left(\lambda_{0}\right)+1,2, \ldots, c_{0}, A\left(\lambda_{0}\right) \in \mathbb{N}$, [4]. The GVMs with $d=d_{0}+c_{0} A\left(\lambda_{0}\right)$ are infinitesimally equivalent to the so-called limits of discrete series [4]. The latter are not related to finite-dimensional representations.

The irreps for $d>d_{0}$ are also called analytic continuation of the discrete series.

In order to be more specific, in the next sections we consider the conformal cases.

6 Note that EHW [4] work with highest weight modules, thus, their ranges are limited from above, while we work with lowest weight modules and our ranges are limited from below. The latter is done to have the intuitive picture of positive energy spectrum bounded from below. There is also a shift of the initial points - in our case $d$ - as energy - is positive, while in the notation of [4] the spectrum includes the point zero. 


\section{Conformal groups}

Let $G=S O_{o}(n, 2)$. We shall consider first the case $n>2$. Then $G$ is the conformal group in $n$-dimensional Minkowski space-time. The Lie algebra, i.e., the conformal algebra $\mathcal{G}=s o(n, 2)$ has three non-trivial non-conjugate parabolic subalgebras $\mathcal{P}_{i}=\mathcal{M}_{i} \oplus \mathcal{A}_{i} \oplus \mathcal{N}_{i}$, $i=0,1,2$, where:

$$
\begin{aligned}
& \mathcal{M}_{0} \cong s o(n-2), \quad \mathcal{A}_{0} \cong s o(1,1) \oplus s o(1,1), \quad \mathcal{N}_{0} \cong \mathbb{R}^{2 n-2} \\
& \mathcal{M}_{1} \cong s o(n-2) \oplus s o(2,1), \quad \mathcal{A}_{1} \cong s o(1,1), \quad \mathcal{N}_{1} \cong \mathbb{R}^{2 n-3} \\
& \mathcal{M}_{2} \cong s o(n-1,1), \quad \mathcal{A}_{2} \cong s o(1,1), \quad \mathcal{N}_{2} \cong \mathbb{R}^{n}
\end{aligned}
$$

The parabolic $\mathcal{P}_{0}$ is a minimal one, and thus is cuspidal. The other two parabolics are maximal, $\mathcal{P}_{1}$ is also cuspidal, while $\mathcal{P}_{2}$ is cuspidal only if $n$ is odd.

We shall use representations induced from the parabolic $\mathcal{P}_{2}$ since the sets of finitedimensional (nonunitary) representations of $\mathcal{M}_{2}$ are in 1-to-1 correspondence with the finite-dimensional (unitary) representations of $s o(n)$ which is the semi-simple subalgebra of the maximal compact subalgebra $\mathcal{K}=s o(n) \oplus s o(2)$. Thus, these induced representations are representations of finite $\mathcal{K}$-type [1]. Relatedly, the number of ERs in the corresponding multiplets is equal to $\left|W\left(\mathcal{G}^{\mathbb{C}}, \mathcal{H}^{\mathbb{C}}\right)\right| /\left|W\left(\mathcal{K}^{\mathbb{C}}, \mathcal{H}^{\mathbb{C}}\right)\right|=2(1+\tilde{h}), \quad \tilde{h} \equiv\left[\frac{n}{2}\right]$, where $\mathcal{H}$ is a Cartan subalgebra of both $\mathcal{G}$ and $\mathcal{K}$. Note also that $\mathcal{K}^{\mathbb{C}} \cong \mathcal{M}_{2}^{\mathbb{C}} \oplus \mathcal{A}_{2}^{\mathbb{C}}$.

The Bruhat decomposition [24] of $\mathcal{G}$ which corresponds to this parabolic is:

$$
\mathcal{G}=\tilde{\mathcal{N}}_{2} \oplus \mathcal{M}_{2} \oplus \mathcal{A}_{2} \oplus \mathcal{N}_{2}
$$

where $\tilde{\mathcal{N}}_{2}=\theta \mathcal{N}_{2},(\theta$ is the Cartan involution in $\mathcal{G})$. The subalgebras in this decomposition have direct physical meaning: $\mathcal{M}_{2}$ is the Lorentz algebra of $n$-dimensional Minkowski space-time $M^{n}$, (the latter differs from $\mathbb{R}^{n}$ by the Lorentzian metric), $\mathcal{N}_{2}$ is the translation algebra of $M^{n}, \mathcal{A}_{2}$ is the subalgebra of dilatations, $\tilde{\mathcal{N}}_{2}$ is the subalgebra of special conformal transformations of $M^{n}$.

We label the signature of the ERs of $\mathcal{G}$ as follows:

$$
\begin{aligned}
\chi= & \left\{n_{1}, \ldots, n_{\tilde{h}} ; c\right\}, \quad n_{j} \in \mathbb{Z} / 2, \quad c=d-\frac{n}{2}, \\
& \left|n_{1}\right|<n_{2}<\cdots<n_{\tilde{h}}, \quad n \text { even }, \\
& 0<n_{1}<n_{2}<\cdots<n_{\tilde{h}}, \quad n \text { odd },
\end{aligned}
$$

where the last entry of $\chi$ labels the characters of $\mathcal{A}_{2}$, and the first $\tilde{h}$ entries are labels of the finite-dimensional nonunitary irreps of $\mathcal{M}_{2}$, (or of the finite-dimensional unitary irrep of $s o(n)$ ), which also fulfil the requirement that the $n_{i}$ 's are either all integer, or all half-integer.

The reason to use the parameter $c$ instead of $d$ is that the parametrization of the ERs in the multiplets is given in a simple intuitive way:

$$
\chi_{1}^{ \pm}=\left\{\epsilon n_{1}, \ldots, n_{\tilde{h}} ; \pm n_{\tilde{h}+1}\right\}, \quad n_{\tilde{h}}<n_{\tilde{h}+1}
$$




$$
\begin{aligned}
\chi_{2}^{ \pm} & =\left\{\epsilon n_{1}, \ldots, n_{\tilde{h}-1}, n_{\tilde{h}+1} ; \pm n_{\tilde{h}}\right\} \\
\chi_{3}^{ \pm} & =\left\{\epsilon n_{1}, \ldots, n_{\tilde{h}-2}, n_{\tilde{h}}, n_{\tilde{h}+1} ; \pm n_{\tilde{h}-1}\right\} \\
& \ldots \\
\chi_{\tilde{h}}^{ \pm} & =\left\{\epsilon n_{1}, n_{3}, \ldots, n_{\tilde{h}}, n_{\tilde{h}+1} ; \pm n_{2}\right\} \\
\chi_{\tilde{h}+1}^{ \pm} & =\left\{\epsilon n_{2}, \ldots, n_{\tilde{h}}, n_{\tilde{h}+1} ; \pm n_{1}\right\} \\
\epsilon & =\left\{\begin{array}{lll} 
\pm, & \text { for } n \text { even } \\
1, & \text { for } n \text { odd }
\end{array}\right.
\end{aligned}
$$

$\left(\epsilon= \pm\right.$ is correlated with $\left.\chi^{ \pm}\right)$.

The ERs in the multiplet are related by intertwining integral and differential operators. The integral operators were introduced by Knapp and Stein [25]. These operators intertwine the pairs $\tilde{\mathcal{C}}_{i}^{+}$and $\tilde{\mathcal{C}}_{i}^{-}$:

$$
G_{i}^{+}: \tilde{\mathcal{C}}_{i}^{-} \longrightarrow \tilde{\mathcal{C}}_{i}^{+}, \quad G_{i}^{-}: \tilde{\mathcal{C}}_{i}^{+} \longrightarrow \tilde{\mathcal{C}}_{i}^{-}, \quad i=1, \ldots, 1+\tilde{h}
$$

Matters are arranged so that in every multiplet only the ER with signature $\chi_{1}^{-}$contains a finite-dimensional nonunitary subrepresentation in a finite-dimensional subspace $\mathcal{E}$. The latter corresponds to the finite-dimensional unitary irrep of $s o(n+2)$ with signature $\left\{n_{1}, \ldots, n_{\tilde{h}}, n_{\tilde{h}+1}\right\}$. The subspace $\mathcal{E}$ is annihilated by the operator $G_{1}^{+}$, and is the image of the operator $G_{1}^{-}$.

Analogously, in every multiplet only the ER with signature $\chi_{1}^{+}$contains holomorphic discrete series representation. In fact, it contains also the conjugate anti-holomorphic discrete series. The direct sum of the holomorphic and the antiholomorphic representations are realized in an invariant subspace $\mathcal{D}$ of the ER $\chi_{1}^{+}$. That subspace is annihilated by the operator $G_{1}^{-}$, and is the image of the operator $G_{1}^{+}$.

Note that the corresponding lowest weight GVM is infinitesimally equivalent only to the holomorphic discrete series, while the conjugate highest weight GVM is infinitesimally equivalent to the anti-holomorphic discrete series.

The intertwining differential operators correspond to non-compact positive roots of the root system of $s o(n+2, \mathscr{C})$, cf. [15]. [In the current context, compact roots of $s o(n+2, \mathbb{C})$ are those that are roots also of the subalgebra $s o(n, \mathbb{C})$, the rest of the roots are noncompact.] Let us denote by $\tilde{\mathcal{C}}_{i}^{ \pm}$the representation space with signature $\chi_{i}^{ \pm}$. The intertwining differential operators act as follows:

$$
\begin{aligned}
& d_{i}: \tilde{\mathcal{C}}_{i}^{-} \longrightarrow \tilde{\mathcal{C}}_{i+1}^{-}, \quad i=1, \ldots, \tilde{h}, \quad \forall n \\
& d_{i}^{\prime}: \tilde{\mathcal{C}}_{i+1}^{+} \longrightarrow \tilde{\mathcal{C}}_{i}^{+}, \quad i=1, \ldots, \tilde{h}, \quad \forall n \\
& d_{\tilde{h}}=d_{\tilde{h}}^{\prime}, \quad n \text { even } \\
& d_{\tilde{h}+1}: \tilde{\mathcal{C}}_{\tilde{h}+1}^{-} \longrightarrow \tilde{\mathcal{C}}_{\tilde{h}}^{+}, \quad n \text { even } \\
& d_{\tilde{h}+1}: \tilde{\mathcal{C}}_{\tilde{h}}^{-} \longrightarrow \tilde{\mathcal{C}}_{\tilde{h}+1}^{+}, \quad n \text { even }
\end{aligned}
$$


The degrees of these intertwining differential operators are given just by the differences of the $c$ entries:

$$
\begin{aligned}
& \operatorname{deg} d_{i}=\operatorname{deg} d_{i}^{\prime}=n_{\tilde{h}+2-i}-n_{\tilde{h}+1-i}, \quad i=1, \ldots, \tilde{h}, \quad \forall n \\
& \operatorname{deg} d_{\tilde{h}+1}=n_{2}+n_{1}, \quad n \text { even }
\end{aligned}
$$

The multiplets can be seen pictorially in $[26] .{ }^{7}$ The equalities between some intertwining differential operators for $n$ even in (3.6) is due to the fact that these operators are produced by singular vectors corresponding to the same positive roots of the root system of $\operatorname{so}(n+$ $2, \mathbb{C})$, cf. [15].

More explicitly, for $n$-even, $n=2 \ell$, the root system of $s o(n+2, \mathscr{C})$ may be given by vectors $e_{i} \pm e_{j}, \quad \ell+1 \geq i>j \geq 1$, where $e_{i}$ form an orthonormal basis in $\mathbb{R}^{\ell+1}$, i.e., $\left(e_{i}, e_{j}\right)=\delta_{i j}$. The non-compact roots may be taken as $e_{\ell+1} \pm e_{i}$. The roots $e_{\ell+1}-e_{i}$, $2 \leq i \leq \ell$, correspond to the operators $d_{\ell+1-i}$, the roots $e_{\ell+1}+e_{i}, 2 \leq i \leq \ell$, correspond to the operators $d_{\ell+1-i}^{\prime}$, the roots $e_{\ell+1} \pm e_{1}$ correspond to the operators $d_{\ell}, d_{\ell}^{\prime}$, resp.

For $n$-odd, $n=2 \ell+1$, the root system of $s o(n+2, \mathscr{C})$ may be given by vectors $e_{i} \pm e_{j}$, $\ell+1 \geq i>j \geq 1, e_{k}, 1 \leq k \leq \ell+1$. The non-compact roots may be taken as $e_{\ell+1} \pm e_{i}$, $e_{\ell+1}$. The roots $e_{\ell+1}-e_{i}, 1 \leq i \leq \ell$, correspond to the operators $d_{\ell+1-i}$, the roots $e_{\ell+1}+e_{i}, 1 \leq i \leq \ell$, correspond to the operators $d_{\ell+1-i}^{\prime}$. The root $e_{\ell+1}$ has a special position, for $n_{i} \in \mathbb{N}$ it corresponds to differential operators of degree $2 n_{i}$ which are degenerations of the integral operators $G_{i}^{+}$. The latter phenomenon is given explicitly for $n=3$ in $[22]$.

Another parametrization of the ER/GVM is by the so-called Dynkin labels. These are defined as follows:

$$
m_{i} \equiv\left(\Lambda+\rho, \alpha_{i}^{\vee}\right)
$$

where $\Lambda=\Lambda(\chi), \quad \rho$ is half the sum of the positive roots of $\mathcal{G}, \alpha_{i}$ denotes the simple roots of $\mathcal{G}, \alpha_{i}^{\vee} \equiv 2 \alpha_{i} /\left(\alpha_{i}, \alpha_{i}\right)$ is the co-root of $\alpha_{i}$. Often it is convenient to consider the so-called Harish-Chandra parameters:

$$
m_{\alpha} \equiv\left(\Lambda+\rho, \alpha^{\vee}\right)
$$

where $\alpha$ is any positive root of $\mathcal{G}$. These parameters are redundant, since obviously they can be expressed in terms of the Dynkin labels, however, as we shall see below, some statements are best formulated in their terms.

The numbers $\operatorname{deg} d_{i}, \operatorname{deg} d_{i}^{\prime}$, are actually part of the Harish-Chandra parameters which correspond to the non-compact positive roots of $s o(n+2, \mathscr{C})$. From these, only $\operatorname{deg} d_{1}$, corresponds to a simple root, i.e., is a Dynkin label.

These labellings will be used in the examples $n=3,4$ below.

Above we restricted to $n>2$. The case $n=2$ is reduced to $n=1$ since $s o(2,2) \cong$ $s o(1,2) \oplus s o(1,2)$. The case $n=1$ is special and is treated separately in the next Section.

7 Actually the diagrams in [26] are for the corresponding Euclidean cases $\operatorname{so}(n+1,1)-$ the diagrams are the same for both signatures. 


\section{4. $\mathrm{SL}(2, \mathrm{R})$}

We start with the conformal case for $n=1: \quad G=S O_{o}(1,2) \cong S L(2, \mathbb{R}) / \mathbb{Z}_{2}$. This is treated separately since $r=1$ and there is only one non-trivial parabolic. Next, the Lie algebra $s o(1,2) \cong s l(2, \mathbb{R})$ is maximally split and the subalgebra $\mathcal{M}$ is trivial. Furthermore, this case is simpler and we can give more details.

\subsection{Discrete series and limits thereof}

We consider $G=S L(2, \mathbb{R})$ following in this Subsection Gelfand et al (the original results are in $[27],[28])$. The elementary representations of $S L(2, \mathbb{R})$ are parametrized by a complex number $s$ and a signature $\epsilon=0,1$. We shall denote the ERs by $D_{\chi}, \chi=[s, \epsilon]$. The discrete unitary series are realized as subspaces of $D_{-s} \equiv D_{\chi}$, with $\chi=[-s, \epsilon=$ $s(\bmod 2)]$, when $s \in \mathbb{Z}_{+}$. More precisely, there are two discrete series UIRs $F_{-s}^{ \pm}$, which are invariant subspaces of $D_{-s}$.

The UIRs realized in $F_{-s}^{+}$nowadays are called holomorphic discrete series, they have a lowest weight vector, the UIRs realized in $F_{-s}^{-}$nowadays are called antiholomorphic discrete series, they have a highest weight vector. Their direct sum $F_{-s}=F_{-s}^{+} \oplus F_{-s}^{-}$is an invariant subspace of $D_{-s}$.

For $s \neq 0$ the Casimir (the infinitesimal character) of $D_{-s}$ is equal to the Casimir of $D_{s}$, which contains as subrepresentation the finite-dimensional (non-unitary) representation $E_{s}$ of $S L(2, \mathbb{R})$ of dimension $s$. The UIRs $F_{0}^{ \pm}$are not related to finite-dimensional representations, correspondingly, they do not fulfil Harish-Chandra's criterion for discrete series, and thus, nowadays they are called limits of discrete series.

For fixed $s \neq 0$ the ERs $D_{-s}$ and $D_{s}$ are partially equivalent, which is realized by two integral operators [later introduced in [25] in general]: $\mathcal{A}_{ \pm s}: D_{\mp s} \longrightarrow D_{ \pm s}$, cf. (3.5). Thus, these two ERs form a doublet. The operator $\mathcal{A}_{s}$ annihilates $F_{-s}$ and its image is $E_{s} \subset D_{s}$. The operator $\mathcal{A}_{-s}$ annihilates $E_{s}$ and its image is $F_{-s}$. Note also that the factor space $F_{s}=D_{s} / E_{s}$ is the direct sum of two further subspaces: $F_{s}=F_{s}^{+} \oplus F_{s}^{-}$, and that the operator $\mathcal{A}_{-s}$ maps $F_{s}^{ \pm}$onto $F_{-s}^{ \pm}$.

For $s=0$ one has: $D_{0}=F_{0}=F_{0}^{+} \oplus F_{0}^{-}$, (setting $\left.E_{0}=0\right)$.

\subsection{Lowest weight representations}

As we mentioned the lowest weight representations are conveniently realized via the lowest weight Verma module $V^{\Lambda}$ over the complexification $\mathcal{G}^{\mathbb{C}}=\operatorname{sl}(2, \mathscr{C})$ of the Lie algebra $\mathcal{G}=s l(2, \mathbb{R})$, and the weight $\Lambda=\Lambda(\chi)=\Lambda(s)$ is determined uniquely by $\chi$.

For $s \in \mathbb{N}$ the Verma module $V^{\Lambda(s)}$ is reducible, it has a finite dimensional factorrepresentation $E_{s}^{+}=V^{\Lambda(s)} / I^{\Lambda(s)}$ of real dimension $s$, (which naturally can be identified with $E_{s}$ from above).

By introducing the conjugation singling out $\mathcal{G}$ we can use the Shapovalov form on $U\left(\mathcal{G}^{+}\right)$, $\left(\mathcal{G}^{+}\right.$are the raising generators of $\left.\mathcal{G}^{\mathscr{C}}\right)$, to define a scalar product in $V^{\Lambda}$ and then positivity produces the list of lowest weight modules.

Using as parameter $d=-s$ the condition for positivity is $d \geq-1$. The point 
$d=-1$ is the first reduction point, which happens in the Verma module $V^{\Lambda(1)}$, cf. above. There the factor-UIR is trivial (being one-dimensional). For $d>-1$ the Verma modules $V^{\Lambda(-d)}$ are irreducible and unitarizable. They are called positive energy representations, the parameter $d$ being the energy or conformal weight. They are also called analytic continuation of the discrete series $F_{-s}^{+}$. In particular, for $d=-s \in \mathbb{I N}$ the Verma module $V^{\Lambda(-d)}$ is infinitesimally equivalent to the holomorphic discrete series irrep $F_{-s}^{+}$, while for $d=0$ it is infinitesimally equivalent to the limit of holomorphic discrete series irrep $F_{0}^{+}$. All this is illustrated in Fig.1. below:

A similar construction exists if we take the conjugate highest weight Verma modules, the role of $F_{-s}^{+}, E_{s}^{+}$being played by $F_{-s}^{-}, E_{s}^{-}$. In that case we are speaking about negative energy representations, with parameter $d=s$, so that we have $d \leq 1$. 


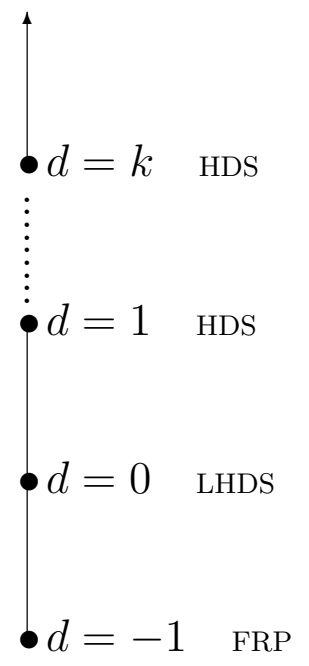

Fig. $1 s l(2, R)$ 


\section{5. $\mathrm{SO}(3,2)$}

The algebra so $(3,2)$ has, besides the minimal parabolic, two maximal (cuspidal) parabolic subalgebras which are isomorphic (though non-conjugate!). So below we fix one of these maximal parabolic subalgebras, and denote it $\mathcal{P}_{\max }$. Here the reducible ERs that have finite $\mathcal{K}$-type representations can be induced from $\mathcal{P}_{\max }=\mathcal{M}_{2} \mathcal{A}_{2} \mathcal{N}_{2}$, where $\mathcal{M}_{2}=$ so $(2,1), \operatorname{dim} \mathcal{A}_{2}=1, \operatorname{dim} \mathcal{N}_{2}=3$. Their signatures are given by $\chi=\left[E_{0}, s_{0}\right]$, where we have introduced the traditionally used energy $E_{0}$ and $\operatorname{spin} s_{0}, s_{0}=0, \frac{1}{2}, 1, \ldots$ (the latter parametrizing the finite-dimensional irreps of $s o(2,1)$ or $s o(3))$.

Alternatively, the ERs (GVMs) are determined by the two Dynkin labels: $m_{i}=$ $\left\langle\Lambda+\rho, \alpha_{i}^{\vee}\right\rangle, \quad i=1,2$, where $\alpha_{i}$ are the simple roots. The relation between the two parametrisations is:

$$
\begin{aligned}
& m_{1}=2 s_{0}+1, \quad m_{2}=1-E_{0}-s_{0}, \\
& \chi=\left[E_{0}, s_{0}\right]=\left[\frac{1}{2}\left(3-m_{1}\right)-m_{2}, \frac{1}{2}\left(m_{1}-1\right)\right]
\end{aligned}
$$

The numbers $m_{3}=m_{1}+2 m_{2}=3-2 E_{0}, m_{4}=m_{1}+m_{2}=2-E_{0}+s_{0}$ correspond to the two non-simple positive roots $\alpha_{3}=\alpha_{1}+\alpha_{2}, \quad \alpha_{4}=2 \alpha_{1}+\alpha_{2}$. The root $\alpha_{1}$ is $\mathcal{M}_{2}$-compact, the other roots are $\mathcal{M}_{2}$-non-compact. The set of the four numbers $m_{i}$, $i=1,2,3,4$, are the Harish-Chandra parameters.

\subsection{Multiplets, finite-dimensional irreps and discrete series}

The reducible such ERs are grouped in quartets, doublets and singlets [22].

The quartets are depicted in Fig. 2, cf. [22]. The signatures of the ERs of the quartet are given by:

$$
\chi_{q, k}^{ \pm}=\left[\frac{1}{2}(3 \pm(q+2 k)), \frac{1}{2}(q-1)\right], \quad \chi_{q, k}^{\prime \pm}=\left[\frac{1}{2}(3 \pm q), \frac{1}{2}(q-1+2 k)\right], \quad q, k \in \mathbb{N}
$$

The quartets are in 1-to-1 correspondence with the finite-dimensional irreps of $G$ since in each quartet there is exactly one ER which contains (as subrepresentation) a finitedimensional irrep. The latter are parametrized by the positive integer Dynkin labels which we denote by $q, k$. The corresponding finite-dimensional irrep is denoted $E_{q, k}$, it has dimension: $q k(q+k)(q+2 k) / 6$, [22], and is contained in the ER denoted by $\chi_{q, k}^{-}$.

Consequently, the quartets also hold the discrete series representations. For fixed $q, k$ the discrete series are contained in $\chi_{q, k}^{+}, \chi_{q, k}^{\prime+}$, - we know that there are two distinct non-conjugate cases of discrete series [6].

The doublets are denoted by $\chi_{q}^{ \pm}$, and the expression for their signatures can be obtained from the signatures of $\chi_{q, k}^{ \pm}$or from $\chi_{q, k}^{\prime \pm}$ by setting $k=0$, i.e.,

$$
\chi_{q}^{ \pm}=\left[\frac{1}{2}(3 \pm q), \frac{1}{2}(q-1)\right], \quad q \in \mathbb{N} .
$$

Note that for all pairs of ERs with signature distinguished by \pm the sum of the $E_{0}$ 's of the two ERs equals 3 - the dimension of Minkowski space-time in this case. Furthermore, 
such pairs are related by two Knapp-Stein integral operators, as in the $S L(2, \mathbb{R})$ case, (though, some of those operators that act from the '-' ER to the ' + ' ER degenerate into differential operators, see below). Thus, each doublet can be represented pictorially by any such \pm pair from the quartet.

The singlets are $\chi_{n}^{s}=\left[\frac{3}{2}, n-\frac{1}{2}\right], n \in \mathbb{N}$, and the expression for their signatures can be obtained from the signatures of $\chi_{q, k}^{ \pm}$by setting $q=2 n, k=-\frac{1}{2} q=-n \quad$ (then \pm coincide). ${ }^{8}$

In Table 1 we give the Harish-Chandra parameters $m_{\alpha}$ for all representations that we discuss in this Section.

According to the results of Harish-Chandra the holomorphic discrete series happen when the numbers $m_{\alpha}$ are negative integers for the $\mathcal{M}_{2}$-non-compact roots. Thus, we see from the Table that the holomorphic discrete series are contained in the ERs $\chi_{q, k}^{+}$.

The limits of the holomorphic discrete series happen when some of the $\mathcal{M}_{2}$-non-compact numbers $m_{\alpha}$ become zero, while the rest of the non-compact numbers $m_{\alpha}$ remain negative. We see that these limits are contained in the ERs $\chi_{q}^{+}$, (from the doublets).

\subsection{Holomorphic discrete series and lowest weight representations}

Next we discuss how the lowest weight positive energy representations fit in the multiplets, and when they are infinitesimally equivalent to holomorphic discrete series.

We are interested in the positive energy UIRs of $\mathcal{G}$ which are given as follows $[29,30,31]$ (with $s_{0} \in \frac{1}{2} \mathbb{Z}_{+}$): ${ }^{9}$

$$
\begin{aligned}
& \text { Rac : } D\left(E_{0}, s_{0}\right)=D(1 / 2,0), \quad \text { Di }: D\left(E_{0}, s_{0}\right)=D(1,1 / 2), \\
& D\left(E_{0} \geq 1, s_{0}=0\right), \quad D\left(E_{0} \geq 3 / 2, \quad s_{0}=1 / 2\right), \quad D\left(E_{0} \geq s_{0}+1, s_{0} \geq 1\right) .
\end{aligned}
$$

The UIRs in (5.4a) are the two singleton representations discovered by Dirac [29] and the last case in $(5.4 b)$ corresponds to the spin- $s_{0}$ massless representations [30]. We note that for these UIRs $m_{2}$ is never a positive integer, $m_{3}$ is a positive integer only for $E_{0}=1 / 2,1$, in which case $m_{3}=2,1$, (Rac,Di), respectively. Similarly, $m_{4}$ is a positive integer only for $E_{0}-s_{0}=1$, and that integer is $m_{4}=1$.

We want to see which positive energy irreps would fit in our multiplets. First we note that no such irreps can fit $\chi_{q, k}^{-}$since $E_{0}=\frac{3}{2}-\frac{1}{2} q-k \leq 0$, and $\chi_{q, k}^{\prime-}$ since $E_{0}=\frac{3}{2}-\frac{1}{2} q \leq 1$ but $s_{0}=\frac{1}{2}(q-1)+k \geq 1$.

We notice that all positive energy irreps from (5.4b) that would fit some multiplet can be parametrized with one parameter $k \in \mathbb{Z}, k \geq-1$. The parametrization is as follows:

$$
m_{1}=q=2 s_{0}+1, \quad m_{2}=-q-k \quad \Longrightarrow \quad E_{0}=2+s_{0}+k
$$

8 Obviously the doublets and singlets are not related to any finite-dimensional representations.

9 We have adopted the notation of [30], so that $D\left(E_{0}, s_{0}\right)$ is the UIR which is contained as subrepresentation of the ER/GVM with signature $\chi=\left[E_{0}, s_{0}\right]$. 
In fact, for $k \geq 1$ we obtain tautologically all ERs $\chi_{q, k}^{+}, q=2 s_{0}+1$, (cf. the Table), which contain all holomorphic discrete series. For $k=0$ we obtain all ERs $\chi_{q}^{+}, q=2 s_{0}+1$, (cf. the Table), which contain all limits of holomorphic discrete series. Finally, for $k=-1$ we obtain the positive energy irreps $D(1,0), D\left(\frac{3}{2}, \frac{1}{2}\right), \quad D\left(s_{0}+1, s_{0}\right)\left(s_{0} \geq 1\right)$, which are contained in the ERs $\chi_{1}^{-}, \chi_{1}^{s}, \chi_{2 s_{0}-1,1}^{\prime+}$.

From this we see that the above would fit the EHW [4] picture with $A\left(\lambda_{0}\right)=1$, (the parameter $z$ from [4] corresponds to our $-k)$. Accordingly the irreps $D(1,0), D\left(\frac{3}{2}, \frac{1}{2}\right)$, $D\left(s_{0}+1, s_{0}\right)\left(s_{0} \geq 1\right)$, are in GVMs which are FRPs. In fact, exceptionally, the UIRs $D(1,0), D\left(\frac{3}{2}, \frac{1}{2}\right)$, are isomorphic to the corresponding GVMs since they happen to be irreducible. ${ }^{10}$

Finally, from the list of positive energy irreps, it remains to discuss the Rac and the Di from $(5.4 a)$. They are found in doublets. With respect to the positive energy spectrum they are isolated points below - by $\frac{1}{2}$-spacing - the FRPs $D(1,0), D\left(\frac{3}{2}, \frac{1}{2}\right)$, resp.

The Rac is in the reducible ER $\chi_{1, \frac{1}{2}}^{-}$. This ER is partially equivalent to the ER $\chi_{1, \frac{1}{2}}^{+}$ (denoted in the Table as Rac*). The latter's GVM is irreducible and also of positive energy: $s_{0}=0, E_{0}=\frac{5}{2}$. The intertwining operator acting from the Rac ER to Rac* is the d'Alembert operator (obtained by reduction of a Knapp-Stein operator [22]).

The Di is in the reducible ER $\chi_{1, \frac{1}{2}}^{\prime-}$. This ER is partially equivalent to the ER $\chi_{1, \frac{1}{2}}^{\prime+}$ (denoted in the Table as $\mathrm{Di}^{*}$ ). The latter's GVM is irreducible and also of positive energy: $s_{0}=\frac{1}{2}, E_{0}=2$.

All this is illustrated on Figures 3,4.

10 Brief explanation: In the case $D(1,0)$ the corresponding Verma module is reducible under roots $\alpha_{1}, \alpha_{3}, \alpha_{4},\left(m_{1}=m_{3}=m_{4}=1\right)$, however, the singular vectors corresponding to the non-compact roots $\alpha_{3}, \alpha_{4}$ turn out to be descendants of the singular vector corresponding to the compact root $\alpha_{1}$. Consequently, when factorizing the Verma module the resulting GVM is irreducible. In the case $D\left(\frac{3}{2}, \frac{1}{2}\right)$ the Verma module is reducible under roots $\alpha_{1}, \alpha_{4},\left(m_{1}=2, m_{4}=1\right)$, however, the singular vector corresponding to the non-compact root $\alpha_{4}$ turns out to be descendant of the singular vector corresponding to the compact root $\alpha_{1}$. See the details in [22]. 


\section{6. $\mathrm{SU}(2,2)$}

In the $4 \mathrm{D}$ conformal case the ERs/GVMs that have finite $\mathcal{K}$-type representations can be induced from the maximal parabolic subalgebra $\mathcal{P}_{\max }=\mathcal{M}_{2} \mathcal{A}_{2} \mathcal{N}_{2}$, where $\mathcal{M}_{2}=$ so(3,1), $\operatorname{dim} \mathcal{A}_{2}=1, \quad \operatorname{dim} \mathcal{N}_{2}=4$. The signatures are given by $\chi=\left[j_{1}, j_{2} ; d\right]$, where $j_{1}, j_{2}$ parametrize the finite-dimensional irreps of $s o(3,1)$, and $d=2+c$ is the energy or conformal weight (while $c$ is parametrizing the $\mathcal{A}_{2}$-character). ${ }^{11}$

Alternatively, the ERs/GVMs are determined by the three Dynkin labels: $m_{i}=\langle\Lambda+$ $\left.\rho, \alpha_{i}^{\vee}\right\rangle$, where $\alpha_{i}$ are the simple roots. The relation between the two parametrisations is:

$$
\begin{aligned}
& m_{1}=2 j_{1}+1, \quad m_{2}=1-d-j_{1}-j_{2}, \quad m_{3}=2 j_{2}+1 \\
& \chi=\left[j_{1}, j_{2} ; d=2+c\right]=\left[\frac{1}{2}\left(m_{1}-1\right), \frac{1}{2}\left(m_{3}-1\right) ; 2-m_{2}-\frac{1}{2}\left(m_{1}+m_{3}\right)\right]
\end{aligned}
$$

The numbers $m_{12}=m_{1}+m_{2}, m_{23}=m_{2}+m_{3}, m_{13}=m_{1}+m_{2}+m_{3}$, correspond to the three non-simple roots in an obvious manner. The roots $\alpha_{1}, \alpha_{3}$ are $\mathcal{M}_{2}$-compact, the other roots are $\mathcal{M}_{2}$-non-compact. The set of the six numbers $m_{\alpha}$ are the Harish-Chandra parameters. We also note that the numbers $m_{1}, m_{3}, c$ parametrize the weights of the maximal compact subalgebra $\mathcal{K}$ and thus, are related to the discrete series representations, see below.

\subsection{Multiplets, finite-dimensional irreps and discrete series}

The reducible ERs/GVMs are grouped in sextets, doublets, and singlets [32],[33]. The sextets are depicted in Fig. 5, cf. [32],[33]. The signatures of the ERs/GVMs of the sextet are given by:

$$
\begin{aligned}
& \chi_{p \nu n}^{-}=\left[\frac{1}{2}(p-1), \frac{1}{2}(n-1) ; 2-\nu-\frac{1}{2}(p+n)\right] \\
& \chi_{p \nu n}^{+}=\left[\frac{1}{2}(n-1), \frac{1}{2}(p-1) ; 2+\nu+\frac{1}{2}(p+n)\right] \\
& \chi_{p \nu n}^{\prime-}=\left[\frac{1}{2}(p+\nu-1), \frac{1}{2}(n+\nu-1) ; 2-\frac{1}{2}(p+n)\right] \\
& \chi_{p \nu n}^{\prime+}=\left[\frac{1}{2}(n+\nu-1), \frac{1}{2}(p+\nu-1) ; 2+\frac{1}{2}(p+n)\right] \\
& \chi_{p \nu n}^{\prime \prime-}=\left[\frac{1}{2}(\nu-1), \frac{1}{2}(p+n+\nu-1) ; 2+\frac{1}{2}(p-n)\right] \\
& \chi_{p \nu n}^{\prime \prime+}=\left[\frac{1}{2}(p+n+\nu-1), \frac{1}{2}(\nu-1) ; 2+\frac{1}{2}(n-p)\right]
\end{aligned}
$$

The sextets are in 1-to- 1 correspondence with the finite-dimensional irreps of $G$ since in each sextet there is exactly one ER/GVM which contains (as subrepresentation) a finitedimensional irrep. The latter are parametrized by the positive integer Dynkin labels which we denote as in [34] by $p, \nu, n \in \mathbb{N}$. Correspondingly, the finite-dimensional irrep is

11 The ERs of the group $G=S U(2,2)$ are induced from the parabolic subgroup $P_{2}=M_{2} A_{2} N_{2}$, cf. (2.1), and the signature of $M_{2} \cong S L(2, \mathbb{C}) \rtimes \mathbb{Z}_{2}$ is given by $\left(j_{1}, j_{2}\right)$ parametrizing the irreps of $s l(2, \mathbb{C}) \cong s o(3,1)$ and the signature $\epsilon=0,1$ parametrizing the character of $\mathbb{Z}_{2}$. In what follows we shall suppress $\epsilon$ since in the multiplet points (see below) it is fixed: $\epsilon=(p+\nu+n-1)(\bmod 2)$, and thus, we shall consider only the Lie algebra picture. 
denoted $E_{p \nu n}$, it has dimension: $p \nu n(p+\nu)(n+\nu)(p+\nu+n) / 12$, [33], and is contained in the ER/GVM denoted by $\chi_{p \nu n}^{-}$.

As a consequence, the sextets also hold the discrete series representations. For fixed $p, \nu, n$ the six representations of the sextet are denoted by: $\chi_{p \nu n}^{ \pm}, \chi_{p \nu n}^{\prime \pm}, \chi_{p \nu n}^{\prime \prime}$. We know that there are three distinct non-conjugate cases of discrete series [6]. In our setting the discrete series are contained in the cases when $c>0: \chi_{p \nu n}^{+}, \chi_{p \nu n}^{\prime+}, \chi_{p \nu n}^{\prime \prime+}(n>p), \chi_{p \nu n}^{\prime \prime}$ $(n<p)$, (the two cases $\chi_{p \nu n}^{\prime \prime \pm}$ are conjugate and count as one case).

The limits of discrete series representations are in some doublets. The doublets are of three kinds, denoted by: ${ }^{1} \chi_{p \nu}^{ \pm},{ }^{2} \chi_{p n}^{ \pm},{ }^{3} \chi_{\nu n}^{ \pm}$, and the expression for their signatures can be obtained from the signatures of $\chi_{p \nu n}^{\prime \pm}$, by setting, $n=0, \nu=0, p=0$, respectively, i.e.,

$$
\begin{aligned}
{ }^{1} \chi_{p \nu}^{\prime-} & =\left[\frac{1}{2}(p+\nu-1), \frac{1}{2}(\nu-1) ; 2-\frac{1}{2} p\right] \\
{ }^{1} \chi_{p \nu}^{\prime+} & =\left[\frac{1}{2}(\nu-1), \frac{1}{2}(p+\nu-1) ; 2+\frac{1}{2} p\right] \\
{ }^{2} \chi_{p n}^{\prime-} & =\left[\frac{1}{2}(p-1), \frac{1}{2}(n-1) ; 2-\frac{1}{2}(p+n)\right] \\
{ }^{2} \chi_{p n}^{\prime+} & =\left[\frac{1}{2}(n-1), \frac{1}{2}(p-1) ; 2+\frac{1}{2}(p+n)\right] \\
{ }^{3} \chi_{\nu n}^{\prime-} & =\left[\frac{1}{2}(\nu-1), \frac{1}{2}(n+\nu-1) ; 2-\frac{1}{2} n\right] \\
{ }^{3} \chi_{\nu n}^{\prime+} & =\left[\frac{1}{2}(n+\nu-1), \frac{1}{2}(\nu-1) ; 2+\frac{1}{2} n\right]
\end{aligned}
$$

Note that for all pairs of ERs/GVMs with signature distinguished by \pm the sum of the conformal weights $d$ of the two ERs/GVMs equals 4 - the dimension of Minkowski spacetime in this case. Furthermore, the ERs of such pairs are related by two Knapp-Stein integral operators [25].

Finally the singlets are denoted by $\chi_{\nu}^{s}=\left[\frac{1}{2}(\nu-1), \frac{1}{2}(\nu-1) ; 2\right],(\nu \in \mathbb{N})$, and the expression for their signatures can be obtained from the signatures of $\chi_{p \nu n}^{\prime \pm}$, by setting, $n=0$ and $p=0$ (then \pm coincide). ${ }^{12}$

In Table 2 we give the Harish-Chandra parameters $m_{\alpha}$ for all representations that we discuss in this Section. ${ }^{13}$

According to the results of Harish-Chandra the holomorphic discrete series happen when the numbers $m_{\alpha}$ are negative integers for the $\mathcal{M}_{2}$-non-compact roots. Thus, we see from the Table that the holomorphic discrete series are contained in the ERs $\chi_{p \nu n}^{+}$. The limits of the holomorphic discrete series happens when some of the non-compact numbers $m_{\alpha}$ become zero, while the rest of the non-compact numbers $m_{\alpha}$ remain negative. We see that these limits are contained in the ERs ${ }^{2} \chi_{p n}^{+}$(obtained also from $\chi_{p \nu n}^{+}$for $\nu=0$ ).

\subsection{Holomorphic discrete series and lowest weight representations}

12 Obviously the doublets and singlets are not related to any finite-dimensional representations.

13 Matters are arranged as discussed so that the Dynkin labels are equal to $p, \nu, n$ for the series $\chi_{p \nu n}^{-}$(which contains the finite-dimensional irreps). 
Next we discuss how the lowest weight positive energy representations fit in the multiplets, and when they are infinitesimally equivalent to holomorphic discrete series.

There are 2 basic cases of positive energy representations [23]:

$$
\text { 1) } j_{1} j_{2} \neq 0, \quad \text { 2) } j_{1} j_{2}=0
$$

In case 1) the positive energy representations fulfil the condition [23]:

$$
d \geq 2+j_{1}+j_{2}, \quad j_{1} j_{2} \neq 0
$$

For $d>2+j_{1}+j_{2}$ the GVMs are irreducible and unitary.

The point $d=d_{0} \equiv 2+j_{1}+j_{2}$ is the first reduction point. In our picture it is realized in the GVM with signature $\chi_{p 1 n}^{\prime+}$, so that $j_{1}=\frac{1}{2} n, j_{2}=\frac{1}{2} p$.

The point $d=d_{0}+1$ is a limit of discrete series, while the integer points with $d \geq$ $d_{0}+2$ are the holomorphic discrete series. Indeed, the former are contained in ${ }^{2} \chi_{p n}^{+}$, while the latter are contained in the ERs with signature $\chi_{p \nu n}^{+}$. In both cases we have $j_{1}=\frac{1}{2}(n-1), j_{2}=\frac{1}{2}(p-1), d=2+\nu+\frac{1}{2}(n+p)$, where $n, p>1$ and $\nu=0, \nu \in \mathbb{N}$, distinguishes the two cases.

Thus, these cases correspond to $c_{0}=1$ (see above) and $A\left(\lambda_{0}\right)=1$ in the terminology of [4]. Here and below the unitarity parameter $z$ of [4] is related to ours as: $z=-d+$ $d_{0}+A\left(\lambda_{0}\right)$.

In case 2) the positive energy representations fulfil the condition [23]:

$$
d \geq 1+j_{1}+j_{2}, \quad j_{1} j_{2}=0
$$

For $d>1+j_{1}+j_{2}$ the GVMs are irreducible and unitary.

The point $d_{0}^{0}=1+j_{1}+j_{2}$ is the first reduction point. These are the massless representations of $s o(4,2)$.

For $j_{1}+j_{2} \geq 1$ the FRP is realized in the ERs/GVMs with signatures: $\chi_{11 n}^{\prime \prime}$, with $j_{1}=\frac{1}{2}(n+1) \geq 1, \quad j_{2}=0$, and $\chi_{p 11}^{\prime \prime}$, with $j_{1}=0, \quad j_{2}=\frac{1}{2}(p+1) \geq 1$.

For $j_{1}+j_{2}=\frac{1}{2}$ the FRP is realized in the ERs/GVMs with signatures: ${ }^{3} \chi_{11}^{-}$, with $j_{1}=\frac{1}{2}, j_{2}=0$, and ${ }^{1} \chi_{11}^{-}$, with $j_{1}=0, \quad j_{2}=\frac{1}{2} .14$

For $j_{1}=j_{2}=0$ the FRP is realized in the ER with signature: ${ }^{2} \chi_{11}^{-} \cdot{ }^{15}$

14 The two conjugated representations are two-component massless Weyl spinors. They are partially equivalent to the ERs ${ }^{3} \chi_{11}^{+},{ }^{1} \chi_{11}^{+}$mentioned below, and the corresponding Knapp-Stein operators from these FRPs degenerate to the two well-known first order conjugated Weyl equations. 15 This massless scalar representation is partially equivalent to the scalar ER ${ }^{2} \chi_{11}^{+}$, mentioned below. The two ERs are related by Knapp-Stein integral operators, however, the operator from ${ }^{2} \chi_{11}^{-}$to ${ }^{2} \chi_{11}^{+}$degenerates to the d'Alembert operator. That d'Alembert operator arises also as 
As we shall see, these cases correspond to $c_{0}=1$ (see above). and $A\left(\lambda_{0}\right)=2$ in the terminology of [4] (the FRP is $z=A\left(\lambda_{0}\right)=2$ ).

The point next to the $\operatorname{FRP}\left(z=1\right.$ by [4]) with $d=d_{0}^{0}+1=2+j_{1}+j_{2}$ is part of the analytic continuation of the discrete series.

For $j_{1}+j_{2} \geq \frac{1}{2}$ it fits the ERs ${ }^{3} \chi_{1 n}^{+}$, so that $j_{1}=\frac{1}{2} n, j_{2}=0$, and ${ }^{1} \chi_{p 1}^{+}$, so that $j_{1}=0$, $j_{2}=\frac{1}{2} p$.

For $j_{1}=j_{2}=0$ it is realized in the singlet ER with signature: $\chi_{1}^{s}$.

The next point with $d=d_{0}^{0}+2=3+j_{1}+j_{2},\left(z=0\right.$ by [4]), fits the ERs ${ }^{2} \chi_{p n}^{+}$ with either $p=1$ or $n=1$, which contain limits of discrete series (with $j_{1}=\frac{1}{2}(n-1)$, $j_{2}=\frac{1}{2}(p-1)$, as above for $\left.j_{1} j_{2} \neq 0\right)$.

Finally, the cases with integer $d \geq d_{0}^{0}+3=4+j_{1}+j_{2} \quad(z<0$ by [4]) are realized by the ERs $\chi_{p \nu n}^{+}$which contain the holomorphic discrete series (as above for $j_{1} j_{2} \neq 0$ ).

All this is illustrated on Figures 6,7.

\subsection{Induction from another parabolic}

The ERs discussed until now can be also induced from the minimal parabolic subgroup as shown in [36], however, then they appear in larger multiplets [33]. In particular, the sextets are part of 24-plets, where each ER corresponds to an element of the Weyl group $W$ of $\mathcal{G}^{\mathbb{C}}=s o\left(6, \mathscr{C}^{\mathcal{C}}\right)$, (recall that $|W|=24$ ).

But besides the minimal and maximal non-cuspidal parabolics (of dimensions 9 and 11, resp.), the group $S U(2,2)$ has a maximal cuspidal parabolic of dimension 10: $\quad P_{1}=$ $M_{1} A_{1} N_{1}$, where $M_{1}=S L(2, \mathbb{R}) \times S O(2), \operatorname{dim} A_{1}=1, \operatorname{dim} N_{1}=5$. The signatures here are $\chi_{1}=\left[n^{\prime}, k, \epsilon, \nu^{\prime}\right]$, where $n^{\prime} \in \mathbb{Z}$ is a character of $S O(2), \nu^{\prime} \in \mathbb{C}$ is a character of $A_{1}, k, \epsilon$ fix a discrete series representation of $S L(2, \mathbb{R}), k \in \mathbb{N}, \epsilon=0,1$, or a limit thereof when $k=0$. In the integer points we are interested in $\epsilon=k(\bmod 2), \nu^{\prime} \in \mathbb{Z}$, and the relation with the Dynkin labels is as follows [33]:

$$
m_{1}=\frac{1}{2}\left(k-\nu^{\prime}+n^{\prime}\right), \quad m_{2}=-k, \quad m_{3}=\frac{1}{2}\left(k-\nu^{\prime}-n^{\prime}\right)
$$

Clearly, representations induced in this way will not describe all ERs with finite K-type, cf. the Table. For instance, one can not obtain the ERs of type $\chi_{p \nu n}^{-}$. Further, there are some restrictions on the values of $n^{\prime}, k, \nu^{\prime}$ when matching the other five series, e.g., to have finite K-types must hold the condition: $k>\nu^{\prime}+\left|n^{\prime}\right|$. Furthermore, in order to fit the holomorphic discrete series, i.e., $\chi_{p \nu n}^{+}$, must hold: $k>\left|\nu^{\prime}\right|+\left|n^{\prime}\right|$. These representations describe the limits of discrete series when $\nu^{\prime}=0$ and $k>\left|n^{\prime}\right|$.

a conditionally invariant differential operator due to the presence of a subsingular vector in the corresponding Verma module with signature $\left(m_{1}, m_{2}, m_{3}\right)=(1,0,1)$ [35]. 


\section{Outlook}

In the present paper we restricted to the conformal group case. Similar explicit descriptions can be easily achieved for the other non-compact groups with lowest/highest weight representations. We plan also to extend these considerations [37] to the supersymmetric cases using precious results on the classification of positive energy irreps in various dimensions $[38],[39],[40],[41]$, and also to the quantum group setting using [42]. Such considerations are expected to be very useful for applications to string theory and integrable models, cf., e.g., $[43]$.

\section{Acknowledgements.}

The author would like to thank for hospitality the International School for Advanced Studies, Trieste, where part of the work was done. This work was supported in part by the Alexander von Humboldt Foundation in the framework of the Clausthal-Leipzig-Sofia Cooperation, and the European RTN 'Forces-Universe', contract MRTN-CT-2004-005104.

The author would like to thank the referees, since their remarks contributed to improving the exposition. 


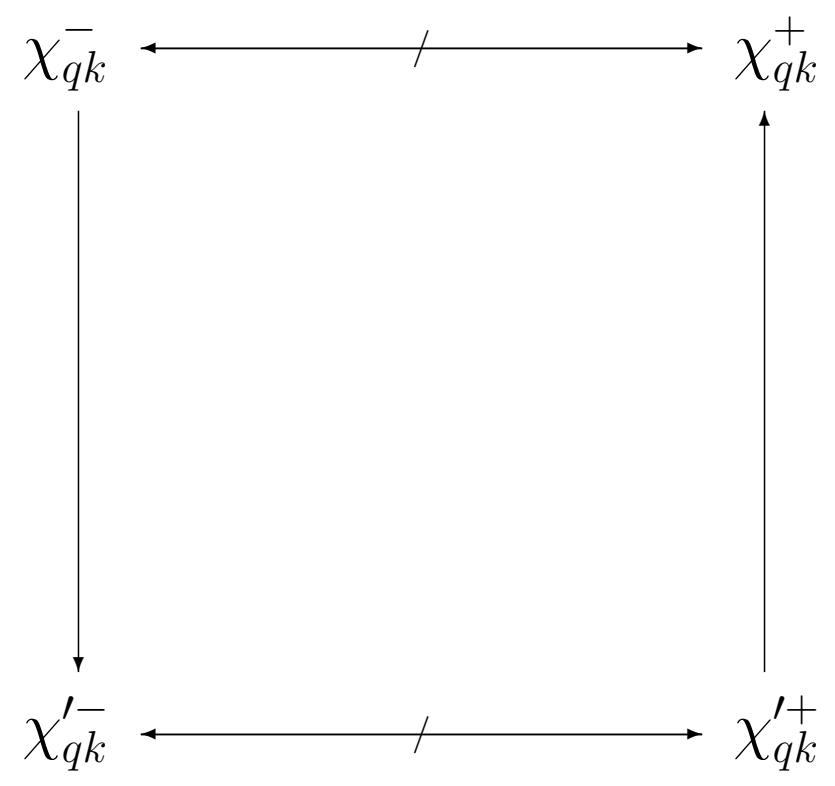

Quartet of partially equivalent ERs and intertwining operators for $s o(3,2)$ and $s o(4,1)$

(arrows are differential operators, dashed arrows are integral operators)

Fig. 2 
Table 1

Harish-Chandra parameters for so(3,2) :

$m_{i}=\left\langle\Lambda+\rho, \alpha_{i}^{\vee}\right\rangle, i=1,2,3,4, m_{3}=m_{1}+2 m_{2}, m_{4}=m_{1}+m_{2}, m_{1} \equiv 2 s_{0}+1$,

\begin{tabular}{|c|c|c|c|c|}
\hline ER & $m_{1}$ & $m_{2}$ & $m_{3}$ & $m_{4}$ \\
\hline$\chi_{q, k}^{-}$ & $q$ & $k$ & $q+2 k$ & $q+k$ \\
\hline$\overline{\chi_{q, k}^{+}}$ & $q$ & $-q-k$ & $-q-2 k$ & $-k$ \\
\hline$\overline{\chi_{q, k}^{\prime-}}$ & $q+2 k$ & $-k$ & $q$ & $q+k$ \\
\hline$\overline{\chi_{q, k}^{\prime+}}$ & $q+2 k$ & $-q-k$ & $-q$ & $k$ \\
\hline$\chi_{q}^{-}$ & $q$ & 0 & $q$ & $q$ \\
\hline$\chi_{q}^{+}$ & $q$ & $-q$ & $-q$ & 0 \\
\hline$\chi_{n}^{s}$ & $2 n$ & $-n$ & $\overline{0}$ & $n$ \\
\hline Rac, $\chi_{1, \frac{1}{2}}^{-}$ & 1 & $\frac{1}{2}$ & 2 & $\frac{3}{2}$ \\
\hline $\operatorname{Rac}^{*}, \chi_{1, \frac{1}{2}}^{+}$ & 1 & $-\frac{3}{2}$ & -2 & $-\frac{1}{2}$ \\
\hline Di, $\chi_{1, \frac{1}{2}}^{\prime-}$ & 2 & $-\frac{1}{2}$ & 1 & $\frac{3}{2}$ \\
\hline $\mathrm{Di}^{*}, \chi_{1, \frac{1}{2}}^{\prime+}$ & 2 & $-\frac{3}{2}$ & -1 & $\frac{1}{2}$ \\
\hline
\end{tabular}




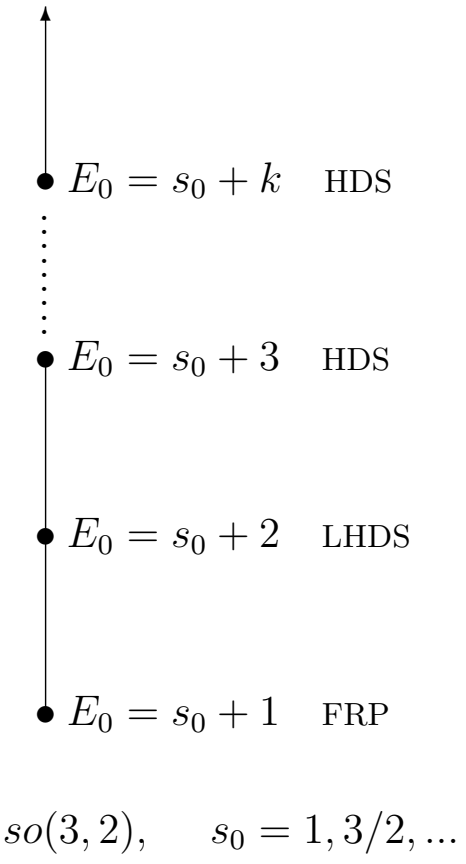

Fig. 3 


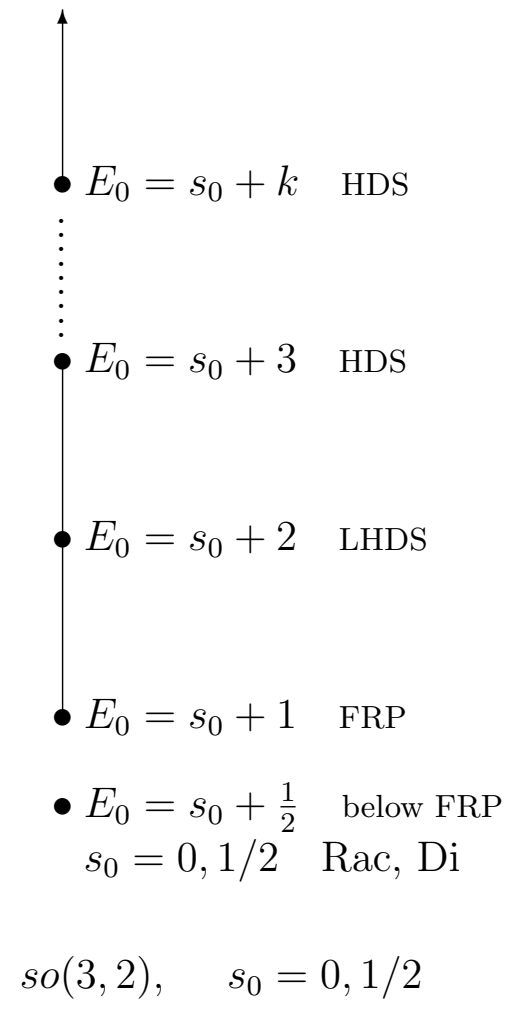

Fig. 4 


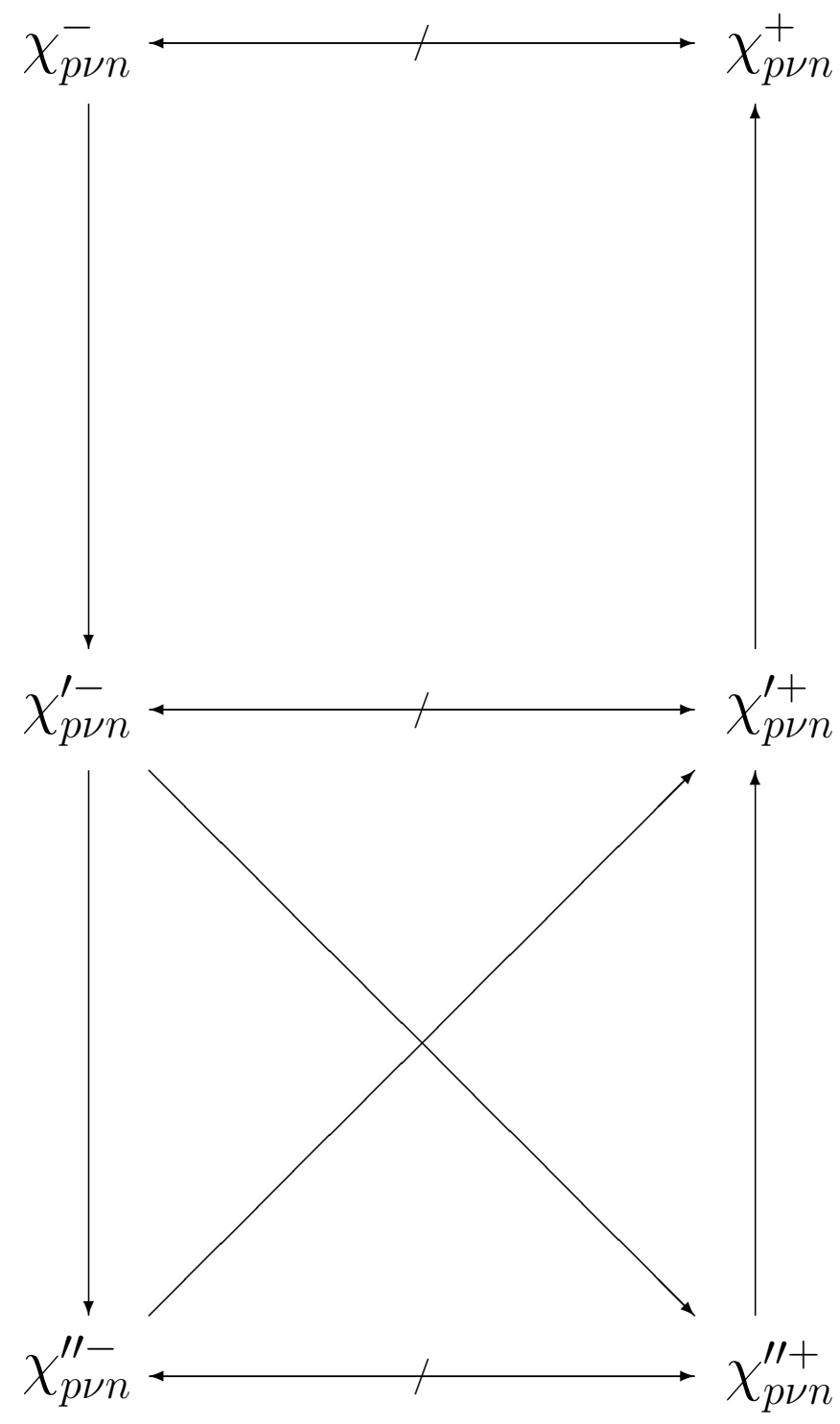

Sextet of partially equivalent ERs and intertwining operators for $s o(4,2)$ and $s o(5,1)$

(arrows are differential operators, dashed arrows are integral operators)

Fig. 5 
Table 2

Harish-Chandra parameters for $\mathbf{s o}(\mathbf{4 , 2})$ :

$m_{i}=\left\langle\Lambda+\rho, \alpha_{i}\right\rangle, i=1,2,3, m_{12}=m_{1}+m_{2}, m_{23}=m_{2}+m_{3}, m_{13}=m_{1}+m_{2}+m_{3}$

\begin{tabular}{|c|c|c|c|c|c|c|}
\hline ER & $m_{1}$ & $m_{2}$ & $m_{3}$ & $m_{12}$ & $m_{23}$ & $m_{13}$ \\
\hline$\chi_{p \nu n}^{-}$ & $p$ & $\nu$ & $n$ & $p+\nu$ & $n+\nu$ & $p+n+\nu$ \\
\hline$\chi_{p \nu n}^{+}$ & $n$ & $-p-\nu-n$ & $p$ & $-p-\nu$ & $-n-\nu$ & $-\nu$ \\
\hline$\chi_{p \nu n}^{\prime-}$ & $p+\nu$ & $-\nu$ & $n+\nu$ & $\bar{p}$ & $n$ & $p+n+\nu$ \\
\hline$\chi_{p \nu n}^{\prime+}$ & $n+\nu$ & $-p-\nu-n$ & $p+\nu$ & $-p$ & $-n$ & $\nu$ \\
\hline$\chi_{p \nu n}^{\prime \prime-}$ & $\nu$ & $-\nu-p$ & $p+n+\nu$ & $-p$ & $n$ & $n+\nu$ \\
\hline$\chi_{p \nu n}^{\prime \prime+}$ & $p+n+\nu$ & $-n-\nu$ & $\nu$ & $p$ & $-n$ & $p+\nu$ \\
\hline${ }^{1} \chi_{p \nu}^{-}$ & $p+\nu$ & $-\nu$ & $\nu$ & $p$ & 0 & $p+\nu$ \\
\hline${ }^{1} \chi_{p \nu}^{+}$ & $\nu$ & $-p-\nu$ & $p+\nu$ & $-p$ & 0 & $\nu$ \\
\hline${ }^{2} \chi_{p n}^{-}$ & $p$ & 0 & $n$ & $p$ & $n$ & $p+n$ \\
\hline${ }^{2} \chi_{p n}^{+}$ & $n$ & $-p-n$ & $p$ & $-p$ & $-n$ & 0 \\
\hline${ }^{3} \chi_{\nu n}^{-}$ & $\nu$ & $-\nu$ & $n+\nu$ & 0 & $n$ & $n+\nu$ \\
\hline${ }^{3} \chi_{\nu n}^{+}$ & $n+\nu$ & $-\nu-n$ & $\nu$ & 0 & $-n$ & $\nu$ \\
\hline$\chi_{\nu}^{s}$ & $\nu$ & $-\nu$ & $\nu$ & 0 & 0 & $\nu$ \\
\hline$P_{n^{\prime}, k, \epsilon, \nu^{\prime}}^{1}$ & $\frac{1}{2}\left(k-\nu^{\prime}+n^{\prime}\right)$ & $-k$ & $\frac{1}{2}\left(k-\nu^{\prime}-n^{\prime}\right)$ & $\frac{1}{2}\left(n^{\prime}-k-\nu^{\prime}\right)$ & $-\frac{1}{2}\left(k+\nu^{\prime}+n^{\prime}\right)$ & $-\nu^{\prime}$ \\
\hline
\end{tabular}




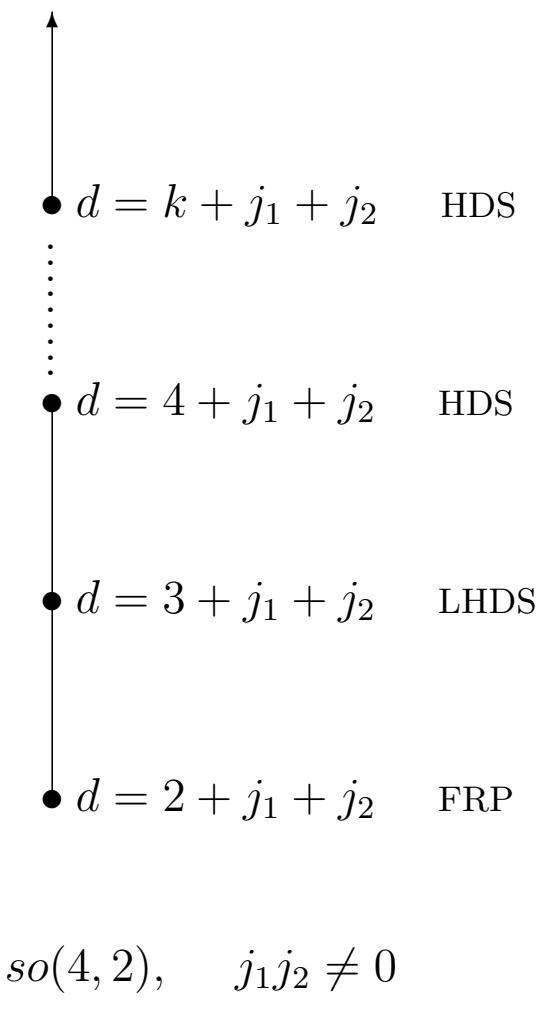

Fig. 6 


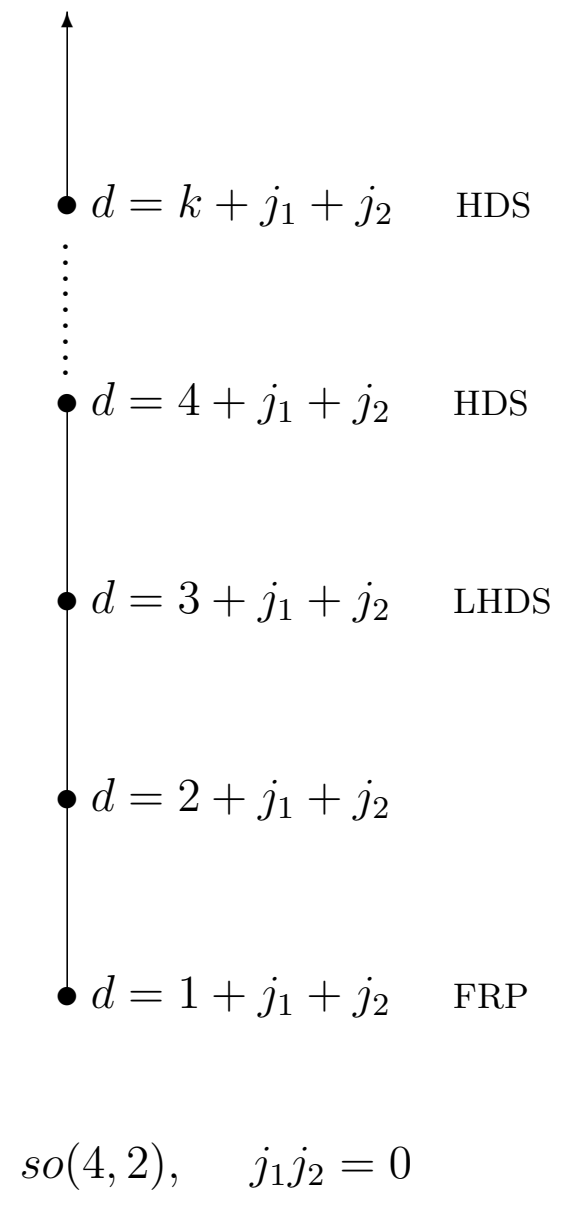

Fig. 7 


\section{References}

[1] Harish-Chandra, "Representations of semisimple Lie groups: IV,V", Am. J. Math. 77 (1955) 743-777, 78 (1956) 1-41.

[2] S. Helgason, Differential Geometry and Symmetric Spaces (Academic Press, New York, 1962).

[3] Harish-Chandra, "Discrete series for semisimple Lie groups: II", Ann. Math. 116 (1966) 1-111.

[4] T. Enright, R. Howe and W. Wallach, "A classification of unitary highest weight modules", in: Representations of Reductive Groups, ed. P. Trombi (Birkhäuser, Boston, 1983) pp. 97-143.

[5] A.W. Knapp and N.R. Wallach, "Szegö kernels associated with discrete series", Invent. Math. 34 (1976) 163-200; "Correction and addition to Szegö kernels associated with discrete series", Invent. Math. 62 (1980) 341-346.

[6] A.W. Knapp, Representation Theory of Semisimple Groups (An Overview Based on Examples), (Princeton Univ. Press, 1986).

[7] B.E. Blank, "Knapp-Wallach Szegö integrals and generalized principal series representations: the parabolic rank one case", J. Funct. Anal. 60 (1985) 127-145; "KnappWallach Szegö integrals. II. The higher parabolic rank case", Trans. Amer. Math. Soc. 300 (1987) 49-59.

[8] M. Kashiwara and M. Vergne, Invent. Math. 44 (1978) 1-47.

[9] N.R. Wallach, Trans. AMS 251 (1979) 1-7 \& 19-37.

[10] R. Parthasarathy, Proc. Indian Acad. Sci. Sect. A Math. Sci. 89 (1980) 124.

[11] H.P. Jakobsen, Invent. Math. 62 (1980) 67-78; Math. Ann. 256 (1981) 439-447; J. Funct. An. 52 (1983) 385-412.

[12] J. Rawnsley, W. Schmid and J.A. Wolf, J. Funct. Anal. 51 (1983) 1114.

[13] L. Dolan, C.R. Nappi and E. Witten, JHEP 0110 (2001) 016, hep-th/0109096.

[14] V.K. Dobrev, Lett. Math. Phys. 9 (1985) 205-211.

[15] V.K. Dobrev, Rep. Math. Phys. 25 (1988) 159-181; first as ICTP Trieste preprint IC/86/393 (1986).

[16] G. Warner, Harmonic Analysis on Semi-Simple Lie Groups I, (Springer, Berlin, 1972).

[17] R.P. Langlands, On the classification of irreducible representations of real algebraic groups, Math. Surveys and Monographs, Vol. 31 (AMS, 1988), first as IAS Princeton preprint (1973).

[18] D.P. Zhelobenko, Harmonic Analysis on Semisimple Complex Lie Groups, (Moscow, Nauka, 1974, in Russian).

[19] A.W. Knapp and G.J. Zuckerman, in: Lecture Notes in Math., Vol. 587 (Springer, Berlin, 1977) pp. 138-159; Ann. Math. 116 (1982) 389-501. 
[20] V.K. Dobrev, G. Mack, V.B. Petkova, S.G. Petrova and I.T. Todorov, Harmonic Analysis on the n-Dimensional Lorentz Group and Its Applications to Conformal Quantum Field Theory, Lecture Notes in Physics, Vol. 63 (Springer-Verlag, Berlin-HeidelbergNew York, 1977).

[21] M. Flato and C. Fronsdal, Lett. Math. Phys. 2, 421-426 (1978); Phys. Lett. B97, 236 (1980); J. Math. Phys. 22 , 1100 (1981); Phys. Rev. D23, 1278 (1981); Phys. Scripta 24, 895 (1981).

[22] V.K. Dobrev, J. Phys. A39 (2006) 5995-6020; hep-th/0512354.

[23] G. Mack, Commun. Math. Phys. 55 (1977) 1.

[24] F. Bruhat, Bull. Soc. Math. France, 84 (1956) 97-205.

[25] A.W. Knapp and E.M. Stein, Ann. Math. 93 (1971) 489-578; II : Inv. Math. 60 (1980) $9-84$.

[26] V.K. Dobrev, Suppl. Rendiconti Circolo Matematici di Palermo, Serie II, Numero 43 (1996) 15-56.

[27] I.M. Gelfand and M.A. Naimark, Acad. Sci. USSR. J. Phys. 10 (1946) 93-94.

[28] V. Bargmann, Annals Math. 48, (1947) 568-640.

[29] P.A.M. Dirac, J. Math. Phys. 4, 901 (1963).

[30] C. Fronsdal, Rev. Mod. Phys. 37, 221 (1965); Phys. Rev. D10, 589 (1974); Phys. Rev. D12, 3819 (1975).

[31] N.T. Evans, J. Math. Phys. 8, 170 (1967).

[32] V.B. Petkova and G.M. Sotkov, Lett. Math. Phys. 8 (1984) 217-226; Erratum-ibid. 9 (1985) 83.

[33] V.K. Dobrev, J. Math. Phys. 26 (1985) 235-251.

[34] V.K. Dobrev and V.B. Petkova, Reports Math. Phys. 13 (1978) 233-277.

[35] V.K. Dobrev, J. Phys. A: Math. Gen. 28 (1995) 7135 - 7155.

[36] V.K. Dobrev and P. Moylan, Fort. d. Phys. 42 (1994) 339-392.

[37] V.K. Dobrev, in preparation.

[38] V.K. Dobrev and V.B. Petkova, Lett. Math. Phys. 9 (1985) 287-298; Fortschr. d. Phys. 35 (1987) 537-572; Phys. Lett. 162B (1985) 127-132.

[39] S. Minwalla, Adv. Theor. Math. Phys. 2 (1998) 781-846, hep-th/9712074.

[40] V.K. Dobrev, J. Phys. A35 (2002) 7079-7100, hep-th/0201076; V.K. Dobrev and R.B. Zhang, Phys. Atom. Nuclei, 68 (2005) 1660-1669, hep-th/0402039; V.K. Dobrev, A.M. Miteva, R.B. Zhang and B.S. Zlatev, Czech. J. Phys. 54 (2004) 1249-1256; hepth/0402056.

[41] C. Carmeli, G. Cassinelli, A. Toigo and V.S. Varadarajan, Comm. Math. Phys. 263 (2006) 217-258, hep-th/0501061.

[42] V.K. Dobrev, Lett. Math. Phys. 22 (1991) 251-266; V.K. Dobrev and P.J. Moylan, Phys. Lett. 315B (1993) 292-298; V.K. Dobrev and R. Floreanini, J. Phys. A: Math. Gen. 27 (1994) 4831-4840; V.K. Dobrev, J. Phys. A: Math. Gen. 27 (1994) 4841-4857 \& 6633-6634, hep-th/9405150.

[43] E. Witten, "Conformal Field Theory in Four and Six Dimensions", arXiv:0712.0157. 